\title{
Betaine attenuates chronic alcohol-induced fatty liver by broadly regulating hepatic lipid metabolism
}

\author{
WENJUAN YANG ${ }^{1}$, LUMING HUANG $^{1}$, JINHANG GAO $^{2}$, SHILEI WEN ${ }^{2}$, YANG TAI ${ }^{1}$, \\ MENG CHEN $^{1}$, ZHIYIN HUANG ${ }^{1}$, RUI LIU ${ }^{2}$, CHENGWEI TANG $^{1,2}$ and JING LI ${ }^{1,2}$ \\ ${ }^{1}$ Department of Gastroenterology, West China Hospital, Sichuan University, Chengdu, Sichuan 610041; ${ }^{2}$ Division of Peptides \\ Related with Human Diseases, State Key Laboratory of Biotherapy, Chengdu, Sichuan 610000, P.R. China
}

Received November 13, 2015; Accepted June 13, 2017

DOI: $10.3892 / \mathrm{mmr} .2017 .7295$

\begin{abstract}
Betaine has previously been demonstrated to protect the liver against alcohol-induced fat accumulation. However, the mechanism through which betaine affects alcohol-induced hepatic lipid metabolic disorders has not been extensively studied. The present study aimed to investigate the effect of betaine on alcoholic simple fatty liver and hepatic lipid metabolism disorders. A total of 36 rats were randomly divided into control, ethanol and ethanol + betaine groups. Liver function, morphological alterations, lipid content and tumor necrosis factor (TNF)- $\alpha$ levels were determined. Hepatic expression levels of diacylglycerol acyltransferase (DGAT) 1, DGAT2, sterol regulatory element binding protein (SREBP)-1c, SREBP-2, fatty acid synthase (FAS), 3-hydroxy-3-methyl-glutaryl (HMG)-CoA reductase, peroxisome proliferator-activated receptor $\lambda$ coactivator (PGC)-1 $\alpha$, adiponectin receptor (AdipoR) 1 and AdipoR2 were quantified. Serum and adipose tissue adiponectin levels were assessed using an enzyme-linked immunoassay. The results demonstrated that alcohol-induced ultramicrostructural alterations in hepatocytes, including the presence of lipid droplets and swollen mitochondria, were attenuated by betaine. Hepatic triglyceride, free fatty acid, total cholesterol and cholesterol ester contents and the expression of DGAT1, DGAT2, SREBP-1c, SREBP-2, FAS and HMG-CoA reductase were increased following ethanol consumption, however were maintained at control levels following betaine supplementation. Alcohol-induced decreases in hepatic PGC-1 $\alpha$ mRNA levels and serum and adipose tissue adiponectin concentrations were prevented by betaine. The downregulation of hepatic AdipoR1 which resulted from alcohol exposure was partially attenuated
\end{abstract}

Correspondence to: Professor Jing Li or Professor Chengwei Tang, Department of Gastroenterology, West China Hospital, Sichuan University, 37 Guoxue Lane, Chengdu, Sichuan 610041, P.R. China E-mail: melody224@163.com

E-mail: shcqcdmed@163.com

Key words: alcoholic fatty liver, betaine, lipids, metabolism, diacylglycerol acyltransferase by betaine. No significant differences in liver function, TNF- $\alpha$, phospholipid and AdipoR 2 levels were observed among the control, ethanol and ethanol + betaine groups. Overall, these results indicated that betaine attenuated the alcoholic simple fatty liver by improving hepatic lipid metabolism via suppression of DGAT1, DGAT2, SREBP-1c, FAS, SREBP-2 and HMG-CoA reductase and upregulation of PGC-1 $\alpha$.

\section{Introduction}

Alcoholic fatty liver is one of the earliest consequences of chronic alcohol consumption (1-4). Excessive lipid accumulation sensitizes the liver to more progressive injuries including steatohepatitis, fibrosis and cirrhosis (5). Although alcohol abstinence is a definitive therapeutic intervention for alcoholic fatty liver, its application is limited by alcohol withdrawal syndrome in alcohol-dependent patients (6). Other than preventing alcohol consumption, the management of alcoholic liver disease only involves treating liver injury present in advanced stages of the disease. No generally accepted medical therapy for alcohol-induced simple fatty liver exists (6). Fortunately, mounting evidence suggests that betaine, additionally termed, trimethyl glycine, acts as a protective agent against alcoholic hepatic steatosis (7-9). However, the mechanism through which betaine affects alcohol-induced hepatic lipid metabolic disorders in the alcoholic simple fatty liver remains to be elucidated.

Increased triglyceride (TG) synthesis is important in the development of the alcoholic fatty liver. This effect of alcohol on TG metabolism was suggested to be partly attributed to the upregulation of diacylglycerol acyltransferase (DGAT) 2, which is a rate-limiting enzyme in TG synthesis (10). Furthermore, the suppression of DGAT2 may be associated with the anti-steatotic effects of betaine in alcoholic fatty liver in mice (11). DGAT1 is another rate-limiting enzyme involved in hepatic TG synthesis, and inhibiting DGAT1 with antisense oligonucleotides or a DGAT-1 inhibitor protects against high-fat diet-induced fatty liver in mice, indicating that DGAT1 may additionally contribute to hepatic steatosis (12-14). However, the role of DGAT1 in the development of alcoholic fatty liver remains to be investigated. The effect of betaine treatment on hepatic DGAT1 expression following alcohol consumption additionally remains to be explored. 
In addition, disturbed fatty acid homeostasis is involved in the pathogenesis of alcoholic fatty liver. It has previously been demonstrated that impaired oxidation and increased synthesis of hepatic fatty acids result from downregulation of peroxisome proliferator-activated receptor $\lambda$ coactivator (PGC)-1 $\alpha$ and the upregulation of sterol regulatory element binding protein (SREBP)-1c and its target gene, fatty acid synthase (FAS), following long-term alcohol intake (15-18). Adiponectin, an adipokine secreted by fat tissue, has been demonstrated to have anti-steatotic properties via the upregulation of PGC-1 $\alpha$ and the suppression of SREBP-1c (4). Alcohol-induced hypoadiponectinemia may result from fatty liver (19). Betaine is a potent agonist of adiponectin and has been demonstrated to prevent the hypoadiponectinmia that results from drinking (19). Therefore, betaine may act as a potential regulator of PGC-1 $\alpha$ and SREBP-1c. However, whether betaine treatment affects hepatic fatty acid homeostasis by regulating the aforementioned key factors remains to be elucidated.

Furthermore, increased cholesterol synthesis is reportedly responsible for hepatic cholesterol accumulation in alcoholic fatty liver (20). The alcohol-induced increase in cholesterol synthesis is closely associated with the activation of hepatic SREBP-2 and its target gene, 3-hydroxy-3-methyl-glutaryl (HMG)-CoA reductase. Betaine has been demonstrated to suppress total cholesterol accumulation in the liver in a steatohepatitis model (21). The effect of betaine on cholesterol metabolism in alcoholic simple fatty liver remains to be verified.

The present study investigated the effect of betaine treatment on alcoholic fatty liver in rats and explored the potential mechanisms underlying the betaine-mediated regulation of hepatic lipid metabolism.

\section{Materials and methods}

Animals and experimental groups. Healthy male Wistar rats (age, 2 months) were purchased from the Experimental Animal Center of Sichuan University (Chengdu, China). The rats were kept individually in cages under a $12 \mathrm{~h}$ light-dark cycle in a temperature $\left(23^{\circ} \mathrm{C}\right)$ - and humidity $(60 \%)$-controlled room with free access to standard rodent chow (28.8\% protein, $6.5 \%$ fat and $64.7 \%$ carbohydrate in total energy) and fluids (as indicated below).

Following 1 week of acclimatization with pure water, 36 rats weighing 210-270 g were randomly divided into 3 groups and given the following as their water source: Water only (control group); ethanol at an initial concentration of $5 \%$ ( $\mathrm{vol} / \mathrm{vol}$ ), which was increased by $5 \%$ weekly up to $25 \%$, and maintained at $25 \%$ for 6 months (ethanol group); or ethanol at the same concentrations plus $1 \%$ betaine [anhydrous; Sigma-Aldrich; Merck KGaA (Darmstadt, Germany); ethanol + betaine group]. Chow and fluid consumption were recorded daily and body weight was recorded weekly.

Upon sacrifice, blood samples were collected from the rat abdominal aortas. The liver and the epididymal fat pads were rapidly collected. The animal experiments in the present study were approved by the Animal Use and Care Committee of Sichuan University and were conducted according to the regulations of Sichuan University.
Serum biochemistry to determine liver function parameters. Serum alanine aminotransferase (ALT), aspartate aminotransferase (AST) and alkaline phosphatase (ALP) were analyzed with an Olympus AU2700 analyzer (Olympus Corporation, Tokyo, Japan) according to standard tests based on the recommendations of the International Federation of Clinical Chemistry (22-24).

Measurement of hepatic and serum lipid content. Liver tissue samples were homogenized in chloroform: Methanol (2:1, vol/vol) and total lipids were extracted via the Folch method (25). Hepatic and serum lipid contents were measured. TG levels were quantified using an automatic biochemical analyzer (Olympus AU5400; Olympus Corporation). Free fatty acid (FFA) contents were determined using an assay kit (colorimetric NEFA kit, Randox Laboratories, Crumlin, Co., Antrim, UK) within $24 \mathrm{~h}$ following sacrifice. Total cholesterol (TC), cholesterol ester (CE) and phospholipid (PL) contents were assessed using commercially available kits (Cholesterol/Cholesteryl Ester Quantitation kit, BioVision Inc., Milpitas, CA, USA; EnzyChrom TM Phospholipid Assay kit, BioAssay Systems, Hayward, CA, USA). The procedures corresponding to the aforementioned kits were all conducted according to the manufacturer's protocol.

Morphological studies of liver steatosis. For hematoxylin and eosin (H\&E) staining, fresh liver tissue was fixed in $4 \%$ paraformaldehyde for $48 \mathrm{~h}$ at room temperature. Then, paraformaldehyde-fixed, paraffin-embedded liver tissue was sectioned at $5 \mu \mathrm{m}$ and stained with hematoxylin $(0.5 \%)$ for $3 \mathrm{~min}$ and with eosin $(0.5 \%)$ for $2 \mathrm{~min}$ at room temperature. For Oil Red O staining, frozen liver tissue was sectioned at $10 \mu \mathrm{m}$ and stained with Oil Red O (1\%, $1 \mathrm{~g} / 100 \mathrm{ml}$ in $70 \%$ ethanol; Ameresco, Inc., Framingham, MA, USA) at $45^{\circ} \mathrm{C}$ for $10 \mathrm{~min}$. The images of $\mathrm{H} \& \mathrm{E}$ and Oil Red $\mathrm{O}$ stained tissue sections were acquired using a BX51 microscope (Olympus Corporation, Tokyo, Japan).

For ultrastructural studies, fresh liver tissue was fixed in $4 \%$ glutaraldehyde ( $\mathrm{pH} \mathrm{7.4)}$ at $4^{\circ} \mathrm{C}$ for $24 \mathrm{~h}$. The glutaraldehyde-fixed tissue was post-fixed in $1 \%$ osmium tetroxide for $1 \mathrm{~h}$ and embedded in Epon 812 resin (Superior Products International II Inc., Shawnee, KS, USA) at $45^{\circ} \mathrm{C}$ overnight and at $60^{\circ} \mathrm{C}$ for $24 \mathrm{~h}$ after dehydration. Subsequently, the embedded tissue was sliced into ultrathin sections $(60 \mathrm{~nm})$ using an LKB III ultramicrotome (Laboratoire Kastler Brossel, Paris, France). The ultrathin sections were stained in uranyl acetate $(1 \%)$ and lead citrate $(3 \%)$ for 15 min respectively at room temperature. The stained ultrathin sections were examined using aHitachi-600 Vtransmission electron microscope (Hitachi, Ltd., Tokyo, Japan).

Enzyme-linked immunosorbent assay (ELISA) to assess adiponectin and tumor necrosis factor (TNF)- $\alpha$ levels. The rat epididymal fat pads and liver samples were homogenized in ice-cold saline (1/10, wt/vol) and then centrifuged at 24,000 x g at $4^{\circ} \mathrm{C}$ for $10 \mathrm{~min}$. The adiponectin concentrations in the supernatant of the epididymal fat pads and serum were quantified with an ELISA kit (Rat Total Adiponectin/Acrp30 Quantikine ELISA kit; cat. no. RRP300; R\&D Systems, Inc., Minneapolis, MN, USA). The TNF- $\alpha$ levels in the liver supernatant was 
measured with a commercially available ELISA kit specific to rat TNF- $\alpha$ (Rat TNF-alpha Quantikine ELISA kit; cat. no. RTA00; R\&D Systems, Inc.). The ELISA procedures were conducted according to the manufacturer's protocol.

Quantification of DGATs, SREBPs and adiponectin receptor (AdipoR) levels with western blotting. Total protein was extracted from frozen liver tissue using a radioimmunoprecipitation assay lysis buffer (Beyotime Institute of Biotechnology, Haimen, China). The protein concentration was determined using a bicinchoninic acid (BCA) protein assay kit (Enhanced BCA Protein Assay kit; Beyotime Institute of Biotechnology). Then, $30 \mu \mathrm{g}$ protein samples were separated using $10 \%$ sodium dodecyl sulfate-polyacrylamide gel electrophoresis, transferred to a polyvinylidene fluoride membrane (EMD Millipore, Billerica, MA, USA), and blocked with $5 \%$ (wt/vol) non-fat dry milk in Tris-buffered saline-containing $0.1 \%$ Tween-20 at room temperature for $2 \mathrm{~h}$. Subsequently, the membrane was incubated with the following primary antibodies: Anti-DGAT1 (goat polyclonal antibody; 1:500; cat. no. ab122924; Abcam, Cambridge, UK), anti-DGAT2 (rabbit polyclonal antibody; 1:500; cat. no. ab96094; Abcam), anti-SREBP-1 (mouse monoclonal antibody; 1:500; cat. no. sc-13551; Santa Cruz Biotechnology, Inc. Dallas, TX, USA), anti-SREBP-2 (rabbit polyclonal antibody; 1:1,000; cat. no. ab28482, Abcam), anti-AdipoR1 (rabbit monoclonal antibody; 1:1,000; cat. no. ab126611; Abcam), anti-AdipoR2 (goat polyclonal antibody; 1:1,000; cat. no. ab77612; Abcam) and anti-glyceraldehyde-3-phosphate dehydrogenase (GAPDH; rabbit polyclonal antibody; 1:1,000; cat. no. AB-P-R 001; Hangzhou Goodhere Biotechnology, Co., Ltd., Hangzhou, China) overnight at $4^{\circ} \mathrm{C}$. Following this, membranes were incubated with horseradish peroxidase-conjugated donkey anti-goat secondary antibody (1:1,000; cat. no. A0181; Beyotime Institute of Biotechnology), goat anti-rabbit secondary antibody $(1: 1,000$; cat. no. A0208; Beyotime Institute of Biotechnology), or goat anti-mouse secondary antibody (1:1,000; cat. no. A0216; Beyotime Institute of Biotechnology) at room temperature for $1 \mathrm{~h}$. The protein bands were visualized using an enhanced chemiluminescence (ECL) detection kit (BeyoECL Plus; Beyotime Institute of Biotechnology) and were exposed to photographic film (Kodak, Rochester, NY, USA). The band densities were determined using Quantity One software, version 4.5.0 (Bio-Rad Laboratories Inc., Hercules, CA, USA). The densities of the target bands were normalized to those of GAPDH. The procedures for the aforementioned kits were conducted according to the manufacturer's protocol.

Hepatic $m R N A$ expression analysis via reverse transcription-quantitative polymerase chain reaction ( $R T-q P C R)$. Total RNA was extracted from the frozen liver tissue using TRIzol ${ }^{\circledR}$ reagent (Invitrogen; Thermo Fisher Scientific, Inc., Waltham, MA, USA). To synthesize the cDNA template, reverse transcription of total RNA samples (5 $\mu \mathrm{g})$ was performed using a first-strand cDNA synthesis kit (Thermo Fisher Scientific, Inc.). RT-qPCR was conducted with the QuantiFast SYBR Green PCR kit (Qiagen GmbH, Hilden, Germany) using the CFX96 real-time PCR detection system (Bio-Rad Laboratories, Inc.). The thermocycling conditions
Table I. Sequences of intron-spanning primers for reverse transcription-quantitative polymerase chain reaction.

\begin{tabular}{lr}
\hline Gene & $\begin{array}{r}\text { Product } \\
\text { size }(b p)\end{array}$ \\
\hline
\end{tabular}

\section{GAPDH}

Forward

TCGGTGTGA ACGGATTTG

Reverse CTCAGC CTTGACTGTGCC

DGAT1

Forward

GCTCCAGCCCATACCCG

Reverse

GCATCACCACGCACCAAT

DGAT2

Forward

GAGACTACTTTCCCATCCAG

Reverse CTTCTTGCTAACTTCGGTG

SREBP-1c

Forward

GCTCACAAAAGCAAATCACT

Reverse

GCGTTTCTACCACTTCAGG

SREBP-2

100

Forward

CAAACACGGAGAAGTAGACAC

Reverse CGGGGAAGATAGGAAGG

FAS

Forward

CTATTGTGGACGGAGGTATC

Reverse TGCTGTAGCCCAGAAGAG

HMG-CoA R

Forward

AAGGTGGTGAGAGAAGTATT

Reverse ATGGCAGTGACGATGTT

PGC- $1 \alpha$

Forward

ACAAGACTATTGAGCGAACC

Reverse

TGGCTTTATGAGGAGGAG

AdipoR1

Forward

Reverse

GGCTGAAAGACAATGACTACC

AdipoR2

Forward

AGCACAAAACCAAGCAAAT

Reverse

TCTTTCTACTGTAACCCAC ACACTCCTGCTCTGACC

GAPDH, glyceraldehyde-3-phosphate dehydrogenase; DGAT, diacylglycerol acyltransferase; SREBP, sterol regulatory element binding protein; FAS, fatty acid synthase; HMG-CoA R, 3-hydroxy-3-methyl-glutaryl-CoA reductase; PGC-1 $\alpha$, peroxisome proliferator-activated receptor $\lambda$ coactivator- $1 \alpha$; AdipoR, adiponectin receptor.

used were as follows: Initial denaturation at $95^{\circ} \mathrm{C}$ for $10 \mathrm{sec}$, followed by combined annealing and extension at $60^{\circ} \mathrm{C}$ for $30 \mathrm{sec}$, for a total of 40 cycles. The mRNA expression of the target genes was normalized to that of GAPDH using the $2^{-\Delta \Delta \mathrm{Cq}}$ method (26). The sequences of the intron-spanning primers are presented in Table I. The procedures for the aforementioned kits were conducted according to the manufacturer's protocol.

Immunohistochemical analyses for hepatic SREBPS and AdipoRs. Liver paraffin sections (5 $\mu \mathrm{m}$ thick) were 
Table II. Parameters of rats in the three groups.

\begin{tabular}{lccc}
\hline Parameters (mean $\pm \mathrm{SD})$ & Control & Ethanol & Ethanol + Betaine \\
\hline Initial body weight $(\mathrm{g})$ & $246.56 \pm 23.38$ & $247.14 \pm 22.86$ & $245.86 \pm 23.15$ \\
Body weight gain $(\%)$ & $133.52 \pm 26.46$ & $130.79 \pm 24.52$ & $131.35 \pm 28.54$ \\
Daily caloric intake (kcal/day) & $88.65 \pm 7.21$ & $89.76 \pm 6.78$ & $90.34 \pm 7.26$ \\
Daily alcohol $(25 \%, \mathrm{vol} / \mathrm{vol})$ intake $(\mathrm{ml})$ & 0 & $34.51 \pm 4.97$ & $34.38 \pm 5.25$ \\
ALT $(\mathrm{U} / \mathrm{l})$ & $66.32 \pm 21.10$ & $52.18 \pm 15.94$ & $60.50 \pm 23.73$ \\
AST $(\mathrm{U} / \mathrm{l})$ & $151.18 \pm 39.44$ & $135.24 \pm 34.94$ & $148.22 \pm 47.98$ \\
ALP $(\mathrm{U} / \mathrm{l})$ & $99.93 \pm 37.11$ & $93.52 \pm 25.74$ & $100.42 \pm 29.41$ \\
Liver TNF- $\alpha$ level $(\mathrm{ng} / \mathrm{mg}$ protein) & $7.29 \pm 1.39$ & $7.24 \pm 2.03$ & $7.19 \pm 1.76$ \\
\hline
\end{tabular}

SD, standard deviation; ALT, alanine transferase; AST, aspartate aminotransferase; ALP, alkaline phosphatase; TNF- $\alpha$, tumor necrosis factor- $\alpha$.

deparaffinized with dimethylbenzene and then rehydrated using graded ethanol. The antigens were retrieved by heating the sections in $10 \mathrm{mmol} / \mathrm{l}$ sodium citrate buffer at $95^{\circ} \mathrm{C}$ for 15 min. Following blocking of the nonspecific immunoreactivity with $5 \%$ bovine serum albumin (Thermo Fisher Scientific, Inc.) at $37^{\circ} \mathrm{C}$ for $45 \mathrm{~min}$, the sections were incubated with the following primary antibodies: Anti-SREBP-1 (1:100), anti-SREBP-2 (1:200), anti-AdipoR1 (1:200) and anti-AdipoR2 (1:200) antibodies at $4^{\circ} \mathrm{C}$ overnight, followed by incubation with the following secondary antibodies: Rabbit anti-goat (cat. no. PV-9003; OriGene Technologies, Inc., Beijing, China), goat anti-rabbit (cat. no. PV-9001; OriGene Technologies, Inc.), or goat anti-mouse (cat. no. PV-9002; OriGene Technologies, Inc.) at $37^{\circ} \mathrm{C}$ for $30 \mathrm{~min}$. Subsequently, the sections were stained with 3,3'-diaminobenzidine $(0.05 \%)$ for $3 \mathrm{~min}$. Sections were counterstained with hematoxylin $(0.5 \%)$ at room temperature for $1 \mathrm{~min}$. For the negative controls, nonimmune serum (Gibco; Thermo Fisher Scientific, Inc.) was used instead of the primary antibodies. Sections were observed under a BX51 microscope (Olympus Corporation) and photomicrographs were captured. The areas positive for SREBP-1c, SREBP-2, AdipoR1 and AdipoR2 were quantified using Image-Pro Plus software version 4.0 (Media Cybernetics, Inc., Rockville, MD, USA), to score the integrated optical density at magnification, x400.

Statistical analysis. The data were expressed as the mean \pm standard deviation of 3 independent experiments and were analyzed using SPSS software, version 13.0 (SPSS, Inc., Chicago, IL, USA). All data were analyzed via one-way analysis of variance followed by the Student-Newman-Keuls test. $\mathrm{P}<0.05$ was considered to indicate a statistically significant difference.

\section{Results}

Betaine does not alter nutritional state, alcohol consumption, liver function or hepatic TNF- $\alpha$ levels. The initial body weight, body weight gain and daily caloric intake of the control, ethanol and ethanol + betaine groups were not significantly different from each other $(\mathrm{P}>0.05)$. The daily alcohol intake was similar for the ethanol group and the ethanol + betaine group $(\mathrm{P}>0.05)$. No differences in serum ALT, AST and
ALP concentrations and hepatic TNF- $\alpha$ levels were observed among the control, ethanol and ethanol + betaine groups (P>0.05; Table II).

Betaine attenuates alcohol-induced alterations in liver morphology. H\&E- and Oil Red O-stained sections demonstrated accumulations of lipid droplets in the livers of the ethanol-fed rats. Ultramicrostructural analyses further revealed that typical lipid droplets and swollen mitochondria were present in the hepatocytes of the rats in the ethanol group. Conversely, lipid droplets and swollen mitochondria were rare in the livers of the control rats. Furthermore, these ethanol-elicited morphological alterations were attenuated in the livers of the rats in the ethanol + betaine group compared with the livers of the ethanol-fed rats. In addition, no fibrosis, biliary dilation, or inflammatory cell infiltration were visualized in the liver tissues of the rats in the control, ethanol and ethanol + betaine groups (Fig. 1).

Betaine decreases alcohol-induced increases in liver lipid content. Compared with the control group, the ethanol group had significantly elevated hepatic TG, FFA, TC and CE levels $(\mathrm{P}<0.05)$. Furthermore, the TG, FFA, TC and CE concentrations in the livers of the rats in the ethanol + betaine group were markedly reduced compared with the ethanol group and were similar to those in the controls. However, there was no significant difference in the hepatic PL content among the control, ethanol and ethanol + betaine groups (Table III).

Betaine exhibits varied effects on alcohol-induced alterations in liver DGAT and PGC-1 $\alpha$ expression. The mRNA and protein expression of DGAT1 and DGAT2 in the liver were enhanced in the ethanol group compared with the controls $(\mathrm{P}<0.05)$. Furthermore, the expression of DGAT1 and DGAT2 was significantly reduced in the ethanol + betaine group compared with the ethanol group and were maintained at the control levels. Compared with the control group, the ethanol group demonstrated markedly downregulated hepatic PGC-1 $\alpha$ mRNA expression $(\mathrm{P}<0.01)$; in contrast, hepatic PGC-1 $\alpha$ mRNA expression was enhanced in the ethanol + betaine group compared with the ethanol group and was similar to that in the control (Fig. 2). In addition to this, hepatic FAS and $\mathrm{HMG}-\mathrm{CoA}$ reductase mRNA expression levels were 
Table III. Selected lipid contents in the liver.

\begin{tabular}{lrrr}
\hline Parameters (mean \pm SD) & Control & Ethanol & Ethanol + Betaine \\
\hline Liver TG content $(\mathrm{mg} / \mathrm{g}$ tissue) & $7.77 \pm 2.22$ & $14.06 \pm 5.97^{\mathrm{a}}$ & $8.64 \pm 4.54^{\mathrm{c}}$ \\
Liver TC content $(\mathrm{mg} / \mathrm{g}$ tissue) & $3.87 \pm 0.91$ & $5.65 \pm 0.69^{\mathrm{a}}$ & $3.63 \pm 0.57^{\mathrm{c}}$ \\
Liver CE content $(\mathrm{mg} / \mathrm{g}$ tissue) & $0.34 \pm 0.20$ & $1.43 \pm 0.30^{\mathrm{a}}$ & $0.18 \pm 0.13^{\mathrm{c}}$ \\
Liver FFA content $(\mu \mathrm{mol} / \mathrm{g}$ tissue) & $2.33 \pm 0.87$ & $3.20 \pm 0.67^{\mathrm{b}}$ & $2.11 \pm 0.81^{\mathrm{c}}$ \\
Liver PL content $(\mathrm{mg} / \mathrm{g}$ tissue) & $0.76 \pm 0.11$ & $0.88 \pm 0.24$ & $0.90 \pm 0.20$ \\
\hline
\end{tabular}

$\mathrm{SD}$, standard deviation; TG, triglyceride; TC, total cholesterol; CE, cholesterol ester; FFA, free fatty acid; PL, phospholipid. ${ }^{\mathrm{a}} \mathrm{P}<0.01,{ }^{\mathrm{b}} \mathrm{P}<0.05$ vs. control; ${ }^{c} \mathrm{P}<0.01$ vs. ethanol group.
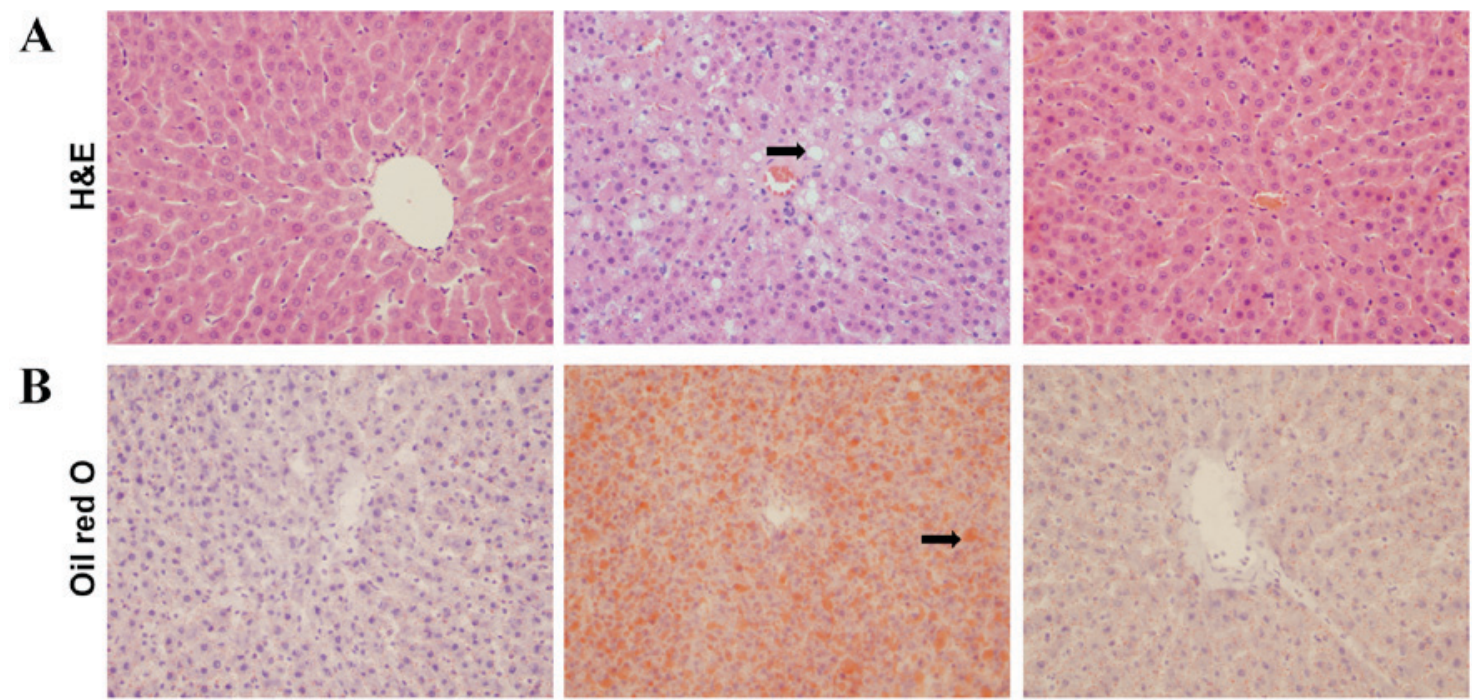

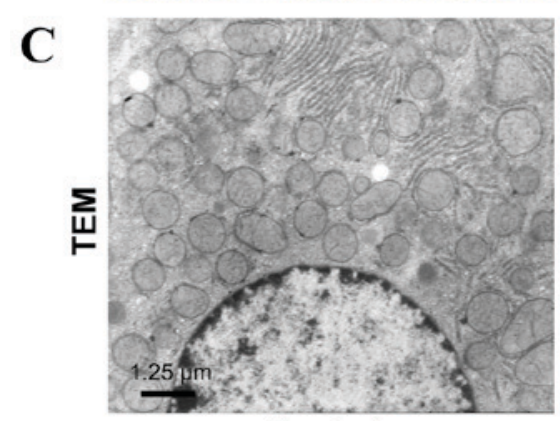

Control

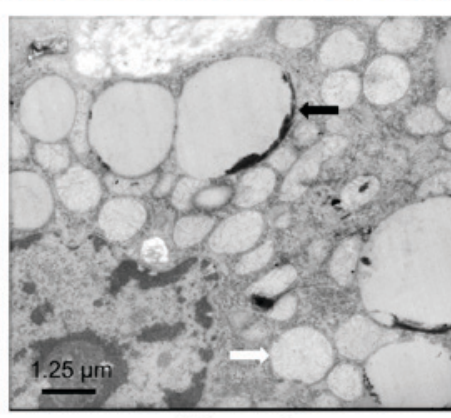

Ethanol

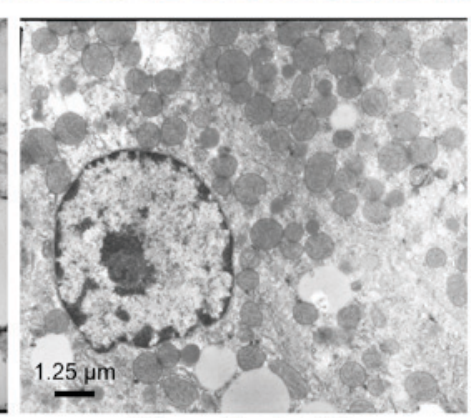

Ethanol + betaine

Figure 1. Morphology of hepatocytes of rats. (A) H\&E and (B) Oil Red O staining (original magnification, $\mathrm{x} 400$ ). (C) Ultrastructural studies (original magnification, x8,000; 1st-2nd panels) or x6,000 (3rd panel). Numerous intracellular lipid droplets (black arrow in A, B and C) and swollen mitochondria (white arrow in C) were present in the hepatocytes of the rats in the ethanol group, whereas these morphological alterations were attenuated in the ethanol + betaine group. $\mathrm{H} \& \mathrm{E}$, hematoxylin and eosin; TEM, transmission electronic microscopy.

significantly increased in the ethanol group compared with the controls and were reduced to similar levels as the controls in the ethanol + betaine group (Fig. 2).

Betaine decreased alcohol-induced SREBP activation in the liver. As presented in the immunohistochemical staining image in Fig. 3, the hepatic expression of SREBP-1c and SREBP-2 (the total expression of the SREBP precursor and mature protein) was markedly upregulated in the ethanol group compared with the controls. Furthermore, the hepatic expression of SREBP-1c and SREBP-2 mRNA and mature protein was significantly increased in the ethanol-fed rats compared with control group $(\mathrm{P}<0.05)$. In addition, with betaine supplementation, the over-expression of hepatic SREBP-1c and SERBP-2 observed in ethanol-fed rats was significantly downregulated to levels of the control group, as demonstrated not only by immunohistochemistry but also in the quantitative western blot and RT-qPCR analyses (Fig. 3).

Betaine increases alcohol-induced alterations in adiponectin and AdipoR1. As presented in Table IV, the serum and epididymal fat pad adiponectin levels in the ethanol group 
A

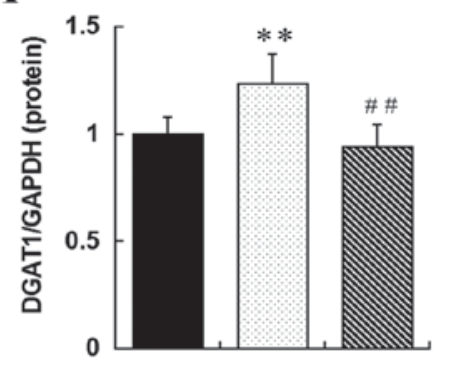

D

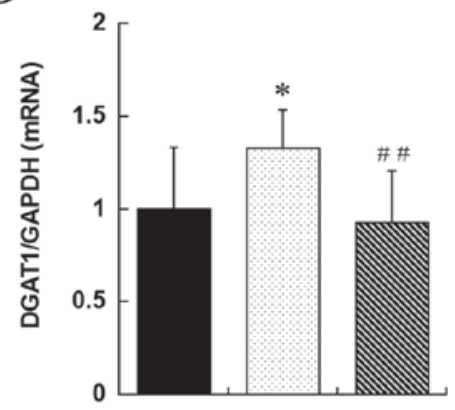

F

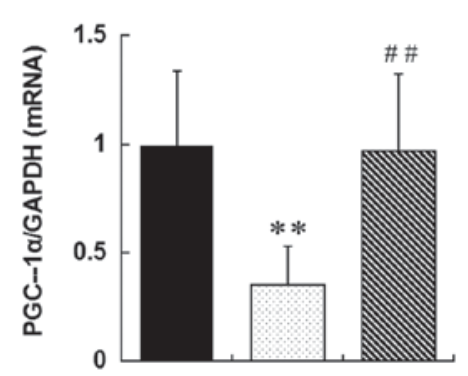

B

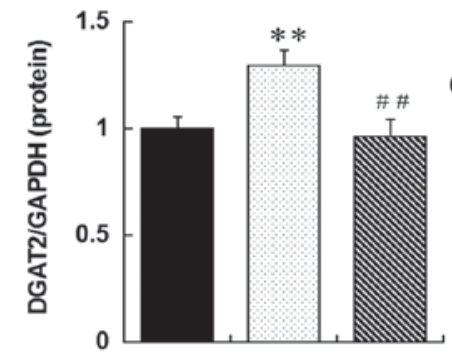

$\mathbf{E}$

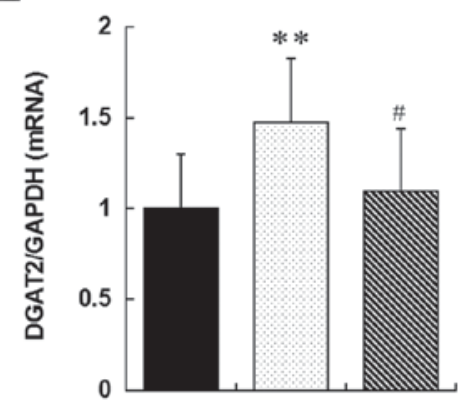

G

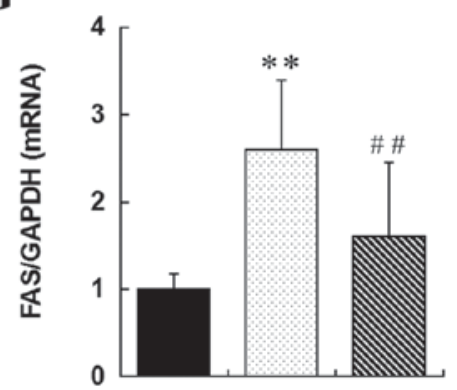

C

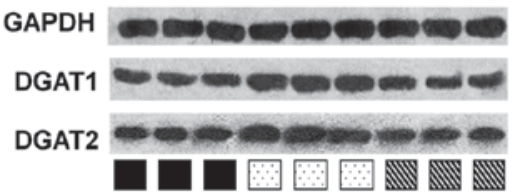

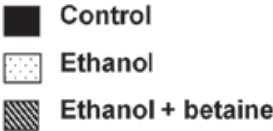

H

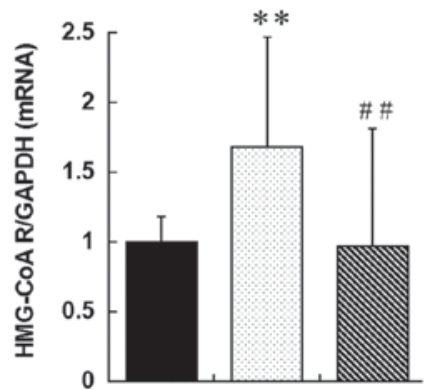

Figure 2. Expression of DGAT1, DGAT2, PGC-1 $\alpha$, FAS and HMG-CoA reductase in the rat liver. Quantitative representation of hepatic protein levels of (A) DGAT1, (B) DGAT2, and (C) representative image, detected by western blotting. Quantitative representation of hepatic mRNA levels of (D) DGAT1 and (E) DGAT2, detected by reverse transcription-quantitative polymerase chain reaction. Quantitative representation of hepatic mRNA levels of (F) PGC-1 $\alpha$, (G) FAS and (H) HMG-CoA reductase, detected by RT-qPCR. ${ }^{* *} \mathrm{P}<0.01,{ }^{*} \mathrm{P}<0.05$ vs. control group; ${ }^{\# /} \mathrm{P}<0.01$, ${ }^{*} \mathrm{P}<0.05$ vs. ethanol group. DGAT, diacylglycerol acyltransferase; PGC-1 $\alpha$, peroxisome proliferator-activated receptor $\lambda$ coactivator-1 $\alpha$; FAS, fatty acid synthase; HMG-CoA reductase, 3-hydroxy-3-methyl-glutaryl-CoA reductase.

were significantly reduced compared with the controls, and these levels were increased to those of the controls in the ethanol + betaine group (Table IV).

As demonstrated via immunohistochemical staining (Fig. 4), hepatic AdipoR1 expression was significantly downregulated in the ethanol group compared with the control and was upregulated in the ethanol + betaine group. Consistent with these results, hepatic AdipoR1 mRNA and protein expression was markedly reduced in the ethanol group compared with the controls $(\mathrm{P}<0.01)$. Furthermore, hepatic AdipoR1 mRNA expression in the ethanol + betaine group was significantly increased compared with the ethanol group and was sustained at the control level $(\mathrm{P}<0.01)$. However, although hepatic AdipoR1 protein expression in the ethanol + betaine group was increased compared with ethanol group, it was still decreased compared with control. Furthermore, hepatic AdipoR2 expression was similar among the control, ethanol and ethanol + betaine groups, as determined via immunohistochemistry, western blotting and RT-qPCR analyses (P>0.05; Fig. 4).

\section{Discussion}

Consistent with numerous alcohol feeding studies, the present study verified that long-term alcohol consumption results in significant hepatic steatosis (27-29). The voluntary oral ethanol-feeding model used in the present study has previously been established (27-31). The administration of alcohol in drinking water while allowing rats to feed on standard rodent chow ad libitum mimics human behavioral patterns of intermittent alcohol use and alterations in dietary intake $(27,31)$. The rats in the control, ethanol and ethanol + betaine groups exhibited comparable calorie intakes and nutritional status. The alcohol burden was also similar for the ethanol and the ethanol + betaine groups. Furthermore, liver function and TNF- $\alpha$ levels were intact following the ethanol feeding procedure, which indicated that the appearance of hepatic steatosis was without steatohepatitis. Therefore, the administration of ethanol and betaine provides a satisfactory rat model for studying the effects of betaine treatment on alcohol-induced simple fatty liver. 
A
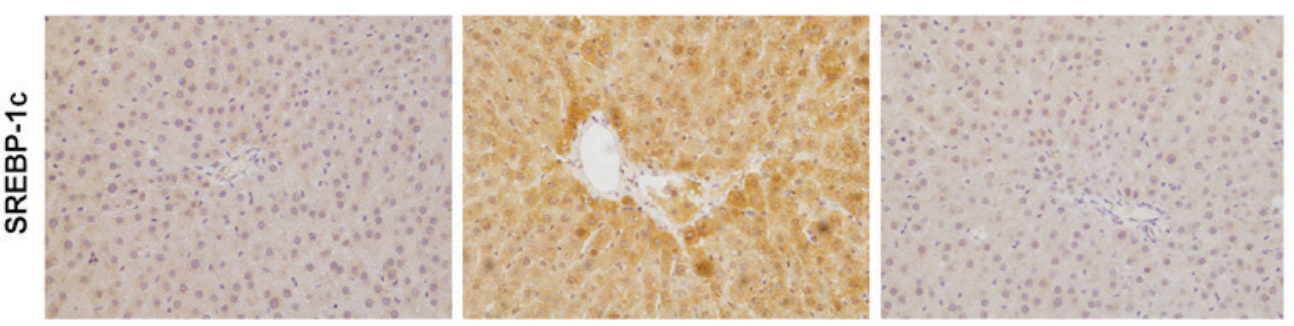

B

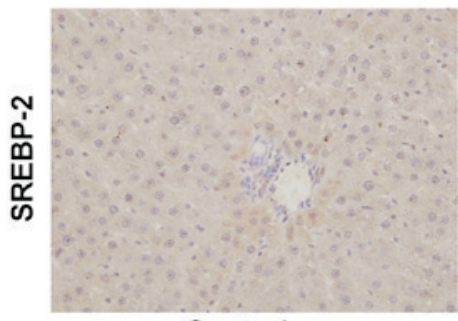

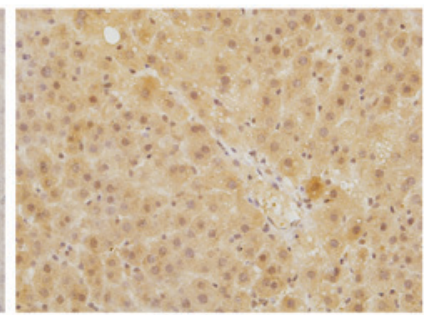

Ethanol

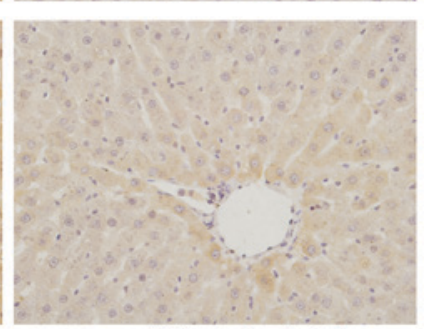

Ethanol + betaine
C

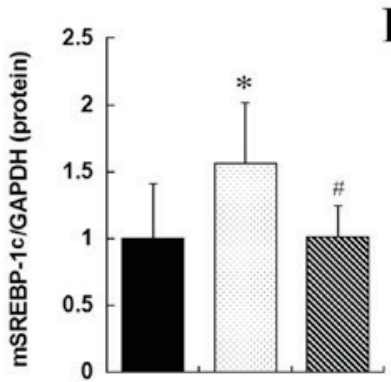

F

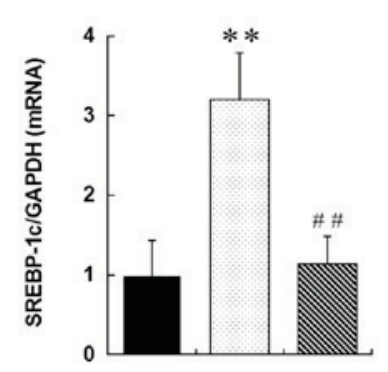

D

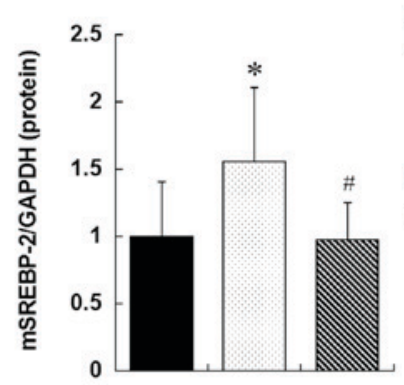

G

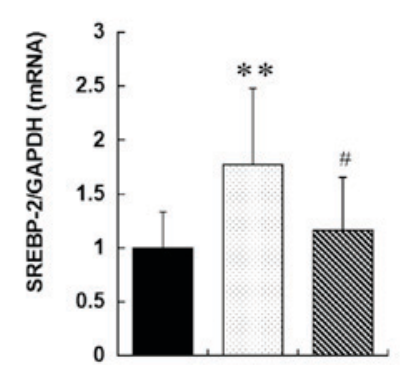

E

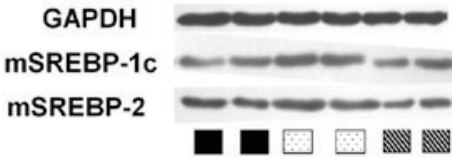

$\square \square \square \mathbb{N}$

Figure 3. Expression of SREBP-1c and SREBP-2 in the rat liver. Immunohistochemical staining images of (A) SREBP-1c and (B) SREBP-2 (original magnification, $x 400$ ). Quantitative representation of hepatic protein levels of (C) mSREBP-1, (D) mSREBP-2 and (E) representative image, detected by western blotting. Hepatic mRNA levels of (F) SREBP-1c and (G) SREBP-2, as detected by reverse transcription quantitative-polymerase chain reaction. ${ }^{* *} \mathrm{P}<0.01$, ${ }^{*} \mathrm{P}<0.05$ vs. control group; ${ }^{\#} \mathrm{P}<0.01,{ }^{,} \mathrm{P}<0.05$ vs. ethanol group. SREBP, sterol regulatory element binding protein; $\mathrm{m}$, mature; GAPDH, glyceraldehyde-3-phosphate dehydrogenase.

Using this rat model, the present study demonstrated that dietary betaine treatment markedly alleviated chronic alcoholic simple fatty liver. The alcohol-induced accumulation of lipid droplets in the liver was attenuated following betaine supplementation. This result is in accordance with those from previous studies $(8,9,32,33)$. Furthermore, the anti-steatotic effect of betaine was revealed to be associated with the suppression of TG, FFA, TC and CE accumulation in the liver, indicating that betaine may exhibit a broad range of effects in alcohol-induced lipid metabolic disorders.

The final step in TG synthesis from three fatty acids and glycerol is catalyzed by the rate-limiting enzymes DGAT1 and DGAT2 (34). In a previous study, betaine was demonstrated to suppress TG synthesis by attenuating DGAT2 upregulation in the alcoholic fatty livers of mice (11). However, the role of DGAT1 in hepatic TG metabolism is additionally important. The present study provides preliminary evidence that chronic alcohol consumption upregulates hepatic DGAT1 in rats. Hepatic DGAT1 overexpression has been demonstrated to result in hepatic TG accumulation in transgenic mice (10), therefore it is conceivable that DGAT1 is important in the development of alcoholic fatty liver by enhancing TG synthesis, as demonstrated in the present study. Furthermore, betaine treatment significantly suppressed hepatic DGAT1 and DGAT2 overexpression in the alcohol-fed rats. These data suggested that DGAT1 and DGAT2 suppression contributed to the betaine-induced hepatic protection against TG accumulation via reduction of TG synthesis in the alcohol-fed rats.

A previous study suggested that ethanol intake enhances fatty acid synthesis by activating SREBP-1c, which upregulates the transcription of lipogenic genes, including FAS (15). Furthermore, alcohol reportedly impairs hepatic fatty acid oxidation by downregulating PGC- $1 \alpha$, which promotes the expression of genes that encode the enzymes involved in mitochondrial fatty acid oxidation by cooperating with peroxisome proliferator-activated receptor (PPAR) $\alpha(16-18)$. 
Table IV. Adiponectin levels in the serum and adipose tissue.

\begin{tabular}{lccc}
\hline Parameters $($ mean $\pm \mathrm{SD})$ & Control & Ethanol & Ethanol + Betaine \\
\hline Serum adiponectin level $(\mathrm{ng} / \mathrm{ml})$ & $13.93 \pm 4.78$ & $4.95 \pm 2.5^{\mathrm{a}}$ & $11.46 \pm 2.90^{\mathrm{b}}$ \\
Adipose adiponectin content $(\mathrm{ng} / \mathrm{g}$ tissue $)$ & $721.93 \pm 296.24$ & $423.16 \pm 226.46^{\mathrm{a}}$ & $693.59 \pm 181.89^{\mathrm{c}}$
\end{tabular}

$\mathrm{SD}$, standard deviation; ${ }^{\mathrm{a}} \mathrm{P}<0.01$ vs. control groups, ${ }^{\mathrm{b}} \mathrm{P}<0.01,{ }^{\mathrm{c}} \mathrm{P}<0.05$ vs. the ethanol group.

A

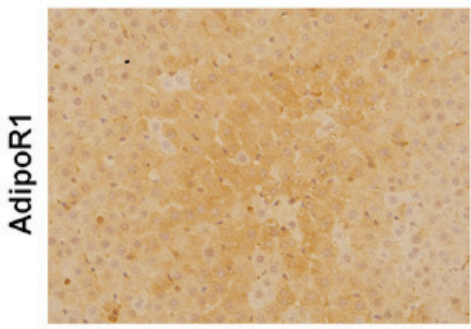

B

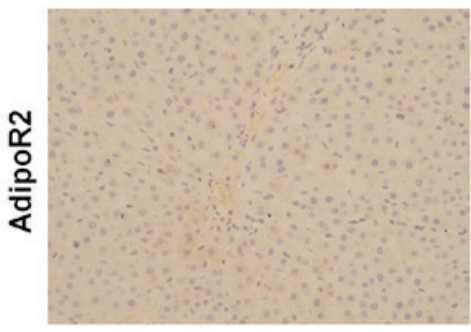

Control

C

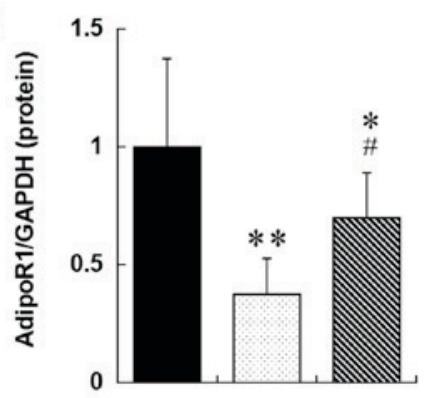

F

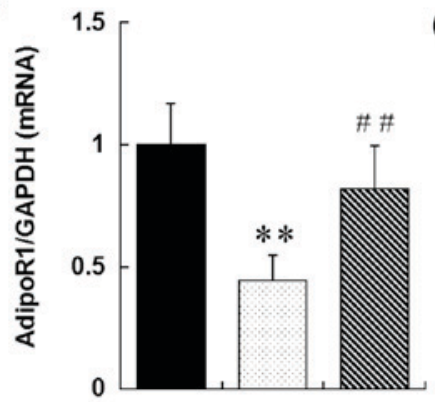

D

G
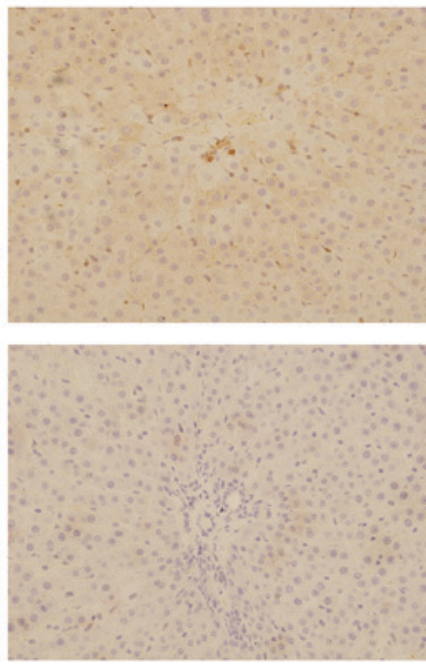

Ethanol

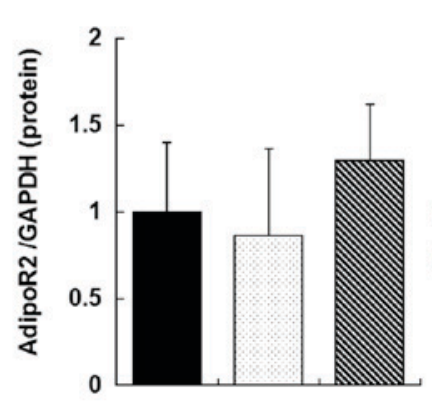

$\mathbf{E}$

GAPDH

AdipoR1

AdipoR2

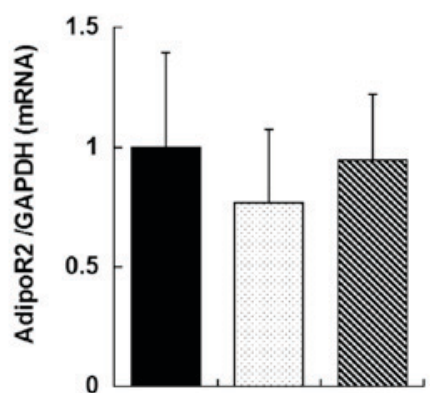

Ethanol + betaine
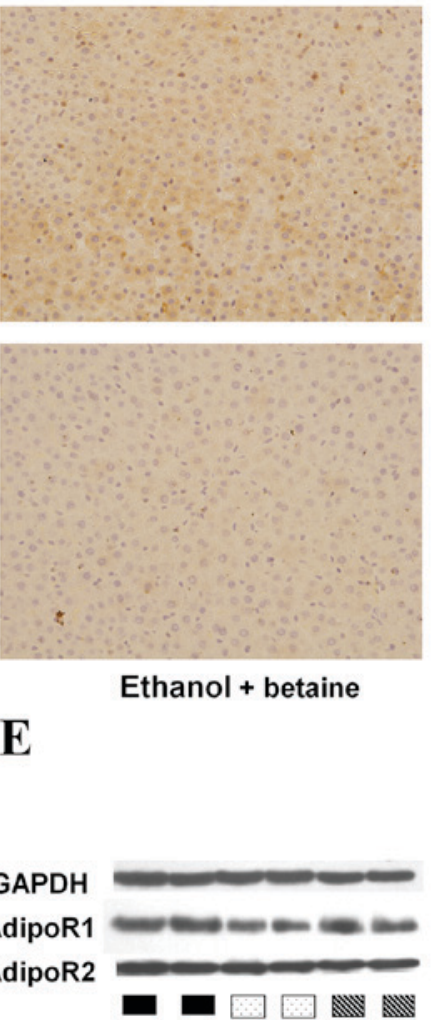

E

- $\square \square \mathbb{N}$

Figure 4. Expression of AdipoR1 and AdipoR2 in the rat liver. Immunohistochemical staining images of (A) AdipoR1 and (B) AdipoR2 (original magnification, x400). Quantitative representation of hepatic protein levels of (C) AdipoR1, (D) AdipoR2 and (E) representative image, as detected by western blotting. Hepatic mRNA levels of (F) AdipoR1 and (G) AdipoR2, as detected by reverse transcription-quantitative polymerase chain reaction. ${ }^{* *} \mathrm{P}<0.01$, ${ }^{*} \mathrm{P}<0.05$ vs. control group; ${ }^{\# \#} \mathrm{P}<0.01,{ }^{\#} \mathrm{P}<0.05$ vs. ethanol group. AdipoR, adiponectin receptor; GAPDH, glyceraldehyde-3-phosphate dehydrogenase.

The results of the present study verified these findings and indicated that alcohol-induced FFA accumulation in the liver was associated with the activation of SREBP-1c-FAS and the suppression of PGC-1 $\alpha$. Furthermore, it was demonstrated that the betaine-induced decrease in hepatic FFA content was concomitant with SREBP-1c and FAS downregulation and PGC-1 $\alpha$ upregulation, which suggested that betaine alleviated alcohol-induced FFA accumulation by correcting an alcohol-induced imbalance of fatty acid synthesis and oxidation by targeting hepatic SREBP-1c-FAS and PGC-1 $\alpha$.

Hypoadiponectinemia results from alcohol-induced SREBP-1c suppression and PGC-1 $\alpha$ upregulation (4). Betaine 
has been demonstrated to restore the serum adiponectin concentration in ethanol-fed rats by protecting adiponectin production in adipose tissue (19). The results of the present study are consistent with these findings. Furthermore, two adiponectin receptors, AdipoR1 and AdipoR2, are expressed in the liver and mediate the effects of adiponectin (35). In the present study, betaine partially attenuated the ethanol-mediated downregulation of hepatic AdipoR1, whereas alcohol and betaine treatment had no effect on hepatic AdipoR2 levels. These results indicated that betaine treatment enhanced hepatic adiponectin signaling in the ethanol-fed rats, although this signaling did not fully recover. Therefore, the betaine-mediated regulation of PGC-1 and SREBP-1c observed in the present study may have resulted in part, from increased hepatic adiponectin signaling.

Furthermore, SREBP-2 is a transcriptional regulator that activates the transcription of genes controlling the synthesis of cholesterol, including HMG-CoA reductase (36). In the present study, alcohol-induced cholesterol accumulation was accompanied by the upregulation of SREBP-2 and HMG-CoA reductase expression in the liver, implying that cholesterol synthesis may be enhanced in the livers of alcohol-fed rats. Furthermore, in a steatohepatitis rat model induced by ethanol plus polyunsaturated fatty acids, betaine was demonstrated to suppress overexpression of SREBP-2 in the liver. The findings verified this in the alcoholic simple hepatic steatosis rat model, and indicated that betaine prevented hepatic cholesterol accumulation potentially by suppressing SREBP-2 and HMG-CoA, and the effects of betaine on cholesterol metabolism were independent of the degree of alcoholic liver disease. In addition, the alterations in hepatic $\mathrm{CE}$ content were in accordance with those of the TC levels observed in the ethanol and ethanol + betaine groups. Therefore, betaine treatment may subsequently suppress CE synthesis by reducing hepatic cholesterol, which is one of the primary raw materials in the production of CE.

There are several limitations in the present study: Only a single dose of betaine was used, and as betaine was administered simultaneously with ethanol, its effects on the reversal of alcoholic fatty liver disease have yet to be elucidated. Therefore, further studies are required to investigate the optimal doses of betaine and confirm its effects on alcoholic fatty liver.

In conclusion, the results of the present study suggested that betaine is a potential medical therapeutic for the alcohol-induced simple fatty liver. Betaine prevented the accumulation of TG, FFA, TC and CE by downregulating DGAT1, DGAT2, SREBP-1c, FAS, SREBP-2 and HMG-CoA reductase and upregulating PGC-1 $\alpha$ in the liver of alcohol-fed rats with simple fatty liver.

\section{Acknowledgements}

The authors would like to thank American Journal Experts for their English language editing service. The present study was supported by the Natural Science Fund of China (grant no. 81170384).

\section{References}

1. Fan JG: Epidemiology of alcoholic and nonalcoholic fatty liver disease in China. J Gastroenterol Hepatol 28 (Suppl 1): S11-S17, 2013.
2. Rehm J, Samokhvalov AV and Shield KD: Global burden of alcoholic liver diseases. J Hepatol 59: 160-168, 2013.

3. O'Shea RS, Dasarathy S and McCullough AJ; Practice Guideline Committee of the American Association for the Study of Liver Diseases; Practice Parameters Committee of the American College of Gastroenterology: Alcoholic liver disease. Hepatology 51: 307-328, 2010.

4. Rogers CQ, Ajmo JM and You M: Adiponectin and alcoholic fatty liver disease. IUBMB Life 60: 790-797, 2008.

5. Yang S, Lin H and Diehl AM: Fatty liver vulnerability to endotoxin-induced damage despite NF-kappaB induction and inhibited caspase 3 activation. Am J Physiol Gastrointest Liver Physiol 281: G382-G392, 2001.

6. European Association for the Study of Liver: EASL clinical practical guidelines: Management of alcoholic liver disease. J Hepatol 57: 399-420, 2012.

7. Barak AJ, Beckenhauer HC, Badakhsh S and Tuma DJ: The effect of betaine in reversing alcoholic steatosis. Alcohol Clin Exp Res 21: 1100-1102, 1997.

8. Balkan J, Oztezcan S, Küçük M, Cevikbaş U, Koçak-Toker N and Uysal M: The effect of betaine treatment on triglyceride levels and oxidative stress in the liver of ethanol-treated guinea pigs. Exp Toxicol Pathol 55: 505-509, 2004.

9. Jung YS, Kim SJ, Kwon dY, Ahn CW, Kim YS, Choi DW and Kim YC: Alleviation of alcoholic liver injury by betaine involves an enhancement of antioxidant defense via regulation of sulfur amino acid metabolism. Food Chem Toxicol 62: 292-298, 2013.

10. Monetti M,Levin MC, Watt MJ, Sajan MP, Marmor S, Hubbard BK, Stevens RD, Bain JR, Newgard CB, Farese RV Sr, et al: Dissociation of hepatic steatosis and insulin resistance in mice overexpressing DGAT in the liver. Cell Metab 6: 69-78, 2007.

11. Wang Z, Yao T and Song Z: Involvement and mechanism of DGAT2 upregulation in the pathogenesis of alcoholic fatty liver disease. J Lipid Res 51: 3158-3165, 2010.

12. Zammit VA: Hepatic triacylglycerol synthesis and secretion: DGAT2 as the link between glycaemia and triglyceridaemia. Biochem J 451: 1-12, 2013.

13. Villanueva CJ, Monetti M, Shih M,Zhou P, Watkins SM, Bhanot S and Farese RV Jr: Specific role for acyl CoA: Diacylglycerol acyltransferase 1 (Dgat1) in hepatic steatosis due to exogenous fatty acids. Hepatology 50: 434-42, 2009.

14. Zhao G, Souers AJ, Voorbach M, Falls HD, Droz B, Brodjian S, Lau YY, Iyengar RR, Gao J, Judd AS, et al: Validation of diacyl glycerolacyltransferase I as a novel target for the treatment of obesity and dyslipidemia using a potent and selective small molecule inhibitor. J Med Chem 51: 380-383, 2008.

15. You M, Fischer M, Deeg MA and Crabb DW: Ethanol induces fatty acid synthesis pathways by activation of sterol regulatory element-binding protein (SREBP). J Biol Chem 277: 29342-29347, 2002.

16. Vega RB, Huss JM and Kelly DP: The coactivator PGC-1 cooperates with peroxisome proliferator-activated receptor alpha in transcriptional control of nuclear genes encoding mitochondrial fatty acid oxidation enzymes. Mol Cell Biol 20: 1868-1876, 2000.

17. Chaung WW, Jacob A, Ji Y and Wang P: Suppression of PGC-1alpha by ethanol: Implications of its role in alcohol induced liver injury. Int J Clin Exp Med 1: 161-170, 2008.

18. Lieber CS, Leo MA, Wang X and Decarli LM: Effect of chronic alcohol consumption on Hepatic SIRT1 and PGC-1alpha in rats. Biochem Biophys Res Commun 370: 44-48, 2008.

19. Song Z, Zhou Z, Deaciuc I, Chen T and McClain CJ: Inhibition of adiponectin production by homocysteine: A potential mechanism for alcoholic liver disease. Hepatology 47: 867-879, 2008.

20. Wang Z, Yao T and Song Z: Chronic alcohol consumption disrupted cholesterol homeostasis in rats: Down-regulation of low-density lipoprotein receptor and enhancement of cholesterol biosynthesis pathway in the liver. Alcohol Clin Exp Res 34: 471-478, 2010.

21. Varatharajalu R, Garige M, Leckey LC, Arellanes-Robledo J, Reyes-Gordillo K, Shah R and Lakshman MR: Adverse signaling of scavenger receptor class B1 and PGC1 s in alcoholic hepatosteatosis and steatohepatitis and protection by betaine in rat. Am J Pathol 184: 2035-2044, 2014.

22. Schumann G, Klauke R, Canalias F, Bossert-Reuther S, Franck PF, Gella FJ, Jørgensen PJ, Kang D, Lessinger JM, Panteghini M and Ceriotti F: IFCC primary reference procedures for the measurement of catalytic activity concentrations of enzymes at $37^{\circ} \mathrm{C}$. Part 9: Reference procedure for the measurement of catalytic concentration of alkaline phosphatase International Federation of Clinical Chemistry and Laboratory Medicine (IFCC) Scientific Division, Committee on Reference Systems of Enzymes (C-RSE) (1)). Clin Chem Lab Med 49: 1439-1446, 2011. 
23. Schumann G, Bonora R, Ceriotti F, Férard G, Ferrero CA Franck PF, Gella FJ, Hoelzel W, Jørgensen PJ, Kanno T, et al: IFCC primary reference procedures for the measurement of catalytic activity concentrations of enzymes at 37 degrees $\mathrm{C}$. International Federation of Clinical Chemistry and Laboratory Medicine. Part 5. Reference procedure for the measurement of catalytic concentration of aspartate aminotransferase. Clin Chem Lab Med 40: 725-733, 2002.

24. Schumann G, Bonora R, Ceriotti F, Férard G, Ferrero CA, Franck PF, Gella FJ, Hoelzel W, Jørgensen PJ, Kanno T, et al: IFCC primary reference procedures for the measurement of catalytic activity concentrations of enzymes at 37 degrees $C$. International Federation of Clinical Chemistry and Laboratory Medicine. Part 4. Reference procedure for the measurement of catalytic concentration of alanine aminotransferase. Clin Chem Lab Med 40: 718-724, 2002.

25. Folch J, Lees M and Sloane Stanley GH: A simple method for the isolation and purification of total lipids from animal tissues. J Biol Chem 226: 497-509, 1957.

26. Livak KJ and Schmittgen TD: Analysis of relative gene expression data using real-time quantitative PCR and the 2(-Delta Delta C(T)) method. Methods 25: 402-408, 2001.

27. Brandon-Warner E, Schrum LW, Schmidt CM and McKillop IH: Rodent models of alcoholic liver disease: Of mice and men. Alcohol 46: 715-725, 2012.

28. Keegan A, Martini R and Batey R: Ethanol-related liver injury in the rat: A model of steatosis, inflammation and pericentral fibrosis. J Hepatol 23: 591-600, 1995.

29. Bujanda L, García-Barcina M, Gutiérrez-de Juan V, Bidaurrazaga J, de Luco MF, Gutiérrez-Stampa M, Larzabal M, Hijona E, Sarasqueta C, Echenique-Elizondo M and Arenas JI: Effect of resveratrol on alcohol-induced mortality and liver lesions in mice. BMC Gastroenterol 6: 35, 2006.
30. Brandon-Warner E, Walling TL, Schrum LW and McKillop IH: Chronic ethanol feeding accelerates hepatocellular carcinoma progression in a sex-dependent manner in a mouse model of hepatocarcinogenesis. Alcohol Clin Exp Res 36: 641-653, 2012.

31. D'Souza El-Guindy NB, Kovacs EJ, De Witte P, Spies C, Littleton JM, de Villiers WJ, Lott AJ, Plackett TP, Lanzke N and Meadows GG: Laboratory models available to study alcohol-induced organ damage and immune variations: Choosing the appropriate model. Alcohol Clin Exp Res 34: 1489-1511, 2010.

32. Ji C and Kaplowitz N: Betaine decreases hyperhomocysteinemia, endoplasmic reticulum stress, and liver injury in alcohol-fed mice. Gastroenterology 124: 1488-1499, 2003.

33. Dou X, Xia Y, Chen J, Qian Y, Li S, Zhang X and Song Z: Rectification of impaired adipose tissue methylation status and lipolytic response contributes to hepatoprotective effect of betaine in a mouse model of alcoholic liver disease. $\mathrm{Br} \mathrm{J}$ Pharmacol 171: 4073-4086, 2014.

34. Farese RV Jr, Cases S and Smith SJ: Triglyceride synthesis: Insights from the cloning of diacylglycerol acyltransferase. Curr Opin Lipidol 11: 229-234, 2000.

35. Kadowaki $\mathrm{T}$ and Yamauchi T: Adiponectin and adiponectin receptors. Endocr Rev 26: 439-451, 2005.

36. Horton JD, Goldstein JL and Brown MS: SREBPs: Activators of the complete program of cholesterol and fatty acid synthesis in the liver. J Clin Invest 109: 1125-1131, 2002. 